\title{
STÉRILISATION DU MATÉRIEL LAITIER
}

\author{
par \\ C. WOLF
}

La question de la stérilisation du matériel laitier est toujours à l'ordre du jour. Elle a été étudiée au cours de l'Assemblée générale de la Société de Technologie laitière (1) de Grande-Bretagne qui s'est tenue à Londres le 10 janvier, sous la Présidence de $R . J$. MacWalter, sous le titre général de : "Stérilisation du matériel laitier avec attention particulière portée au rôle des produits chimiques", étant bien entendu que le terme de "stérilisation" ne s'applique, dans la pratique, qu'à l'élimination sélective de certains groupes de bactéries.

C'est W.A. Cuthbert, chef de la Section bactériologique à l'Institut national de Recherches laitières de Laiterie (N.I.R.D.) de Shinfield, Reading, qui a traité pour débuter de l'évaluation des systèmes pratiqués dans les fermes laitières. Après avoir défini les termes : stérilisation, désinfection et assainissement, il indique que, suivant la définition qu'il en a donnée, la stérilisation ne peut pas être obtenue à la ferme. La désinfection qui consiste à éliminer ou à détruire l'infection, c'est-à-dire à détruire les organismes nuisibles ou indésirables, mais ne détruisant pas nécessairement toutes les formes de vie, lui parait le terme le plus approprié. Dans les premiers temps de la production hygiénique du lait, la chaleur, sous forme de vapeur ou d'eau chaude, était le seul agent acceptable. Au cours des vingt dernières années, il s'est produit beaucoup de changements dans les méthodes de désinfection pratiquées dans les fermes laitières et dans les règlements les concernant. $C$. examine ensuite quelques-unes des méthodes utilisées, le degré de succès obtenu par elles et suggère quelques améliorations à leur utilisation. D'une enquête récente effectuée en Angleterre et dans le Pays de Galles dans environ 1200 fermes, il résulte que la majorité des fermes $(76,5$ p. 100) utilisent des installations de traite mécanique avec seaux désinfectés par des applications rapides de produits chimiques, les agents stérilisants utilisés pour le lavage du matin étant : la vapeur ou l'eau bouillante (14,7 p. 100); l'hypochlorite $(56,1$ p. 100) ; les détersifs-stérilisateurs (27,1 p. 100),

(1) The Journal of the Society of Dairy Technology, vol. XIV, n० 2, avril 1961 , pp. 56-80. 
le nettoyage par immersion n'étant utilisé que dans 2,8 p. 100 des installations $(2,4$ p. 100 avec de la soude caustique et 0,4 p. 100 avec un détersif-stérilisateur). Tous ces systèmes, à l'exception du nettoyage par circulation, qui en est encore au stade expérimental, sont susceptibles de donner des résultats satisfaisants quand ils sont bien appliqués. Malgré cela, le lait est encore de qualité bactériologique insuffisante parce que le matériel est insuffisamment nettoyé et désinfecté et ceci souvent parce que les produits chimiques ne sont pas appliqués à la concentration voulue, ni pendant le temps nécessaire sur des surfaces qui ne sont ni propres ni en bon état et que certains traitements périodiques supplémentaires, qui sont indispensables, ne sont pas pratiqués. Ces traitements supplémentaires peuvent consister pour les parties en caoutchouc en la conservation journalière par trempage dans un liquide ou en un traitement périodique par trempage dans un détersif chaud à $82,2^{\circ}$. Un traitement par- la chaleur (pas moins de $85^{\circ}$ pendant 2 minutes) et le détartrage du matériel métallique peuvent aussi être nécessaires. Le nettoyage par circulation est d'utilisation récente et a besoin d'être encore étudié. Les installations de traite avec tuyauteries ont augmenté en nombre au cours des cinq dernières années de 2 à 7 p. 100 du nombre des producteurs de lait et continueront probablement encore à augmenter. La raison principale pour laquelle les tuyauteries n'avaient pas eu de succès dans le passé, était le coût du nettoyage et surtout le fréquent démontage nécessaire. Le nettoyage sans démontage est maintenant accepté et pratiqué. Les résultats obtenus au début ont été décevants. Les numérations bactériennes étaient élevées, quel que fut le type d'installation. Il ressort des études effectuées qu'il ne faut pas compter uniquement sur la désinfection chimique. Un traitement périodique par la chaleur doit lui être adjoint. Une circulation hebdomadaire d'eau chaude à $76,70_{-} 82,2^{\circ}$ pendant 2 minutes donne de bons résultats; mais ce traitement ne peut être appliqué économiquement que dans les laiteries pour lesquelles il ne faut qu'un petit volume d'eau. En résumé, $C$. est d'avis que la désinfection par la vapeur ou l'eau chaude est entièrement satisfaisante ; mais trop coûteuse si on la compare aux autres systèmes de nettoyage et de désinfection dont les coûts varient de 2,7 à 4,8 p. 100 du coût total de la production du lait, ce qui n'est pas tellement élevé. A noter que les numérations de rincage sont considérées comme non satisfaisantes quand elles dépassent 7750 par centimètre carré. Ce chiffre peut être discuté. Les divers systèmes de nettoyage utilisés ne diffèrent pas énormément au point de vue coût, mais de très grandes différences sont notées de ferme à 
ferme. Le tableau ci-dessous indique le coût annuel du nettoyage en nouveaux francs, la livre étant décomptée à 13,70 NF. :

\begin{tabular}{|c|c|c|c|}
\hline Méthode utilisée & Moyenne & \multicolumn{2}{|c|}{ Limites } \\
\hline Vapeur & $579,510 \mathrm{NF}$ & 278,11 à & $032,295 \mathrm{NF}$ \\
\hline Nettoyage par circulation. . & $493,885 \mathrm{NF}$ & 196,595 à & $813,780 \mathrm{NF}$ \\
\hline $\begin{array}{r}\text { Composé d'ammonium qua- } \\
\text { ternaire } \ldots \ldots \ldots \ldots \ldots\end{array}$ & $457,580 \mathrm{NF}$ & 250,710 à & $758,98 \quad \mathrm{NF}$ \\
\hline Hypechlorite .......... & $441,140 \mathrm{NF}$ & 265,095 à & $841,18 \mathrm{NF}$ \\
\hline Nettoyage par immersion ... & $329,485 \mathrm{NF}$ & 196595 à & $619,925 \mathrm{NF}$ \\
\hline
\end{tabular}

T.R. Ashton, Directeur technique d'une des plus grandes sociétés laitières anglaises a ensuite traité de l' "évaluation des systèmes pratiqués à la réception et dans les centres de traitement \%. Il estime que le nettoyage et la stérilisation ne peuvent pas être considérés comme deux opérations distinctes. La stérilisation commence déjà dans les stades initiaux du nettoyage, pendant le rincage, et est continuée et peut même être complétée quand le lavage est terminé. Il examine ensuite en détail ces diverses opérations. La vapeur est, d'après lui, le procédé le plus efficace de stérilisation; mais elle coûte cher et il est nécessaire d'en limiter l'emploi. La stérilisation à l'eau chaude n'est pas si difficile. Si nécessaire, le rincage, suivant le lavage au détersif, peut être effectué à $85^{\circ}$ ou à une température plus élevée pendant au minimum 10 minutes. Cette façon de faire remplit les exigences à la fois du rincage et de la stérilisation et est une bonne méthode à adopter quand on a utilisé des détersifs non germicides. Quand on a utilisé des détersifs germicides, l'effet germicide diminue progressivement quand la dilution avec de l'eau a lieu, et dès que par un test du rincage, on constate l'absence d'un des principaux constituants chimiques du détersif utilisé, le rincage doit être arrêté. Lorsqu'une laiterie doit être utilisée immédiatement après un rincage à l'eau chaude et une stérilisation combinés, il peut être nécessaire de la refroidir. Dans ce cas, il y a lieu de la rincer à l'eau froide, éventuellement chlorée à 0,2 à 0,5 partie par million de chlore. Si la laiterie n'est pas utilisée à nouveau immédiatement après le traitement, par exemple si elle ne travaille pas la nuit, un rincage final avec une solution froide d'hypochlorite est généralement effectué, Ia force de cette solution pouvant varier de 
20 à 250 à 300 parties par million et la durée de contact variant suivant la capacité et la dimension de l'installation. Normalement, ce traitement est suivi d'un second rincage pour éliminer l'hypochlorite. Il n'est pas habituel d'utiliser des composés d'ammonium quaternaires seuls pour stériliser la laiterie. Ces substances sont souvent combinées avec des détersifs non-ioniques et sont spécialement utiles pour le lavage détersif des installations ayant été en contact avec du lait froid : tuyauteries, remplisseurs, cuves de stockage, etc. La pratique consistant à utiliser de la vapeur ou des solutions concentrées d'hypochlorite avec l'idée qu'elles stérilisent efficacement la laiterie, quel que soit son état, est nettement mauvaise. La surface des souillures peut être stérilisée; mais il existe habituellement de nombreux organismes enrobés ou couverts par ces souillures. Les écheos rencontrés dans l'utilisation des stérilisants chimiques sont dus à l'idée fausse que ces produits en solution concentrée détruisent tout, ce qui est faux lorsque les surfaces à nettoyer ne sont pas propres. En réalité, le nettoyage et la stérilisation sont des opérations inséparables qui, dans la pratique, ont lieu simultanément.

$P$. Dyson (appartenant à une importante société de produits chimiques) a ensuite traité de l'utilisation des composés du chlore. La plupart des hypochlorites contiennent 0,5 p. 100 ou moins d'alcali caustique libre quoique les règlements anglais autorisent un maximum de 2 p. 100 , puis il examine le problème du nettoyage par circulation.

G. A. Thomas (appartenant à la même société de produits chimiques) traite ensuite de l'utilisation des composés d'ammonium quaternaires (C.A.Q.). On peut les considérer comme des dérivés des sels d'ammonium par substitution de groupes organiques aux atomes d'hydrogène dans l'ion ammonium. Quatre types différents de ces composés sont utilisés en Angleterre. L'une de leurs propriétés les plus évidentes est leur activité superficielle réduisant la tension superficielle de l'eau dans laquelle ils sont dissous. Les solutions de C.A.Q. produisent donc de la mousse quand elles sont secouées ou agitées et il n'est pas douteux que cette activité superficielle soit un facteur contribuant à leur activité anti-bactérienne. Ils sont pratiquement sans odeur et sans couleur et ne sont pas toxiques aux concentrations recommandées pour leur utilisation, ni corrosives pour les surfaces en métal inoxydable. Ils sont extrêmement stables, ne montrant aucune perte d'activité après stockage prolongé, et leurs solutions peuvent être utilisées aussi chaudes que nécessaires sans perte d'effet bactéricide. Certains C.A.Q. possèdent 
à la fois des propriétés bactéricides et bactériostatiques, détruisant activement les bactéries aux concentrations élevées, et inhibant leur croissance sans nécessairement les détruire aux concentrations plus faibles. On les utilise généralement à la concentration de 200 parties par million; mais ils ont encore une activité bactériostatique aux concentrations de 10 à 20 parties par million. On dit quelquefois que les C.A.Q. ne sont pas efficaces contre les bactéries Gram-négatives. Aux concentrations utilisées normalement, les C.A.Q. sont aussi activement bactéricides contre les organismes Gram-négatifs; mais, comme beaucoup de produits chimiques stérilisants, ils ne sont pas particulièrement efficaces contre les spores bactériennes dans les conditions normales d'utilisation. Leur activité bactérienne peut être aussi influencée par le type d'eau utilisée, l'eau dure tendant à gêner leur activité. Quelquesuns sont néanmoins plus résistants aux effets de l'eau dure, et, en tout cas, cet effet de l'eau dure peut être modifié par l'utilisation d'alcalis convenables conjointement avec le C.A.Q. La matière organique tend aussi à réduire l'action bactéricide des C.A.Q., comme c'est le cas avec tous les stérilisants chimiques; mais les C.A.Q. sont moins gênés, à cet égard, que beaucoup d'autres produits chimiques. Il est impossible, dans la pratique, de détruire à 100 p. 100 tous les types de micro-organismes existant à la laiterie par l'utilisation des C.A.Q., mais les C.A.Q. réduisent leur nombre à un point tel que leur survie n'a pas d'importance au point de vue hygiénique. Les Américains utilisent, du reste, pour définir ce résultat, non pas le mot "stérilisation ", mais celui de "sanitisation " (assainissement) qui commence aussi à être utilisé en Angleterre. La méthode générale d'utilisation des C.A.Q. est la suivante : $1^{\circ}$ Rincage de l'installation et du matériel à l'eau propre froide ou tiède; $2^{\circ}$ Traitement de l'installation et du matériel avec une solution chaude d'un détersif convenable. En laiterie, on utilise généralement des détersifs alcalins; $3^{\circ}$ Traitement des surfaces avec une solution de C.A.Q. de concentration convenable pendant une période de temps convenable; $4^{\circ}$ Rincage à l'eau propre. Il existe une tendance à combiner les stades $2^{\circ}$ et $3^{\circ}$ par l'utilisation d'un stérilisateur-détersif et de nombreuses préparations contenant du C.A.Q. sont mises en vente à cet effet qui donnent des résultats satisfaisants; mais cette méthode n'est pas toujours à recommander. En résumé, d'après T., les avantages principaux des C.A.Q. en laiterie sont leur absence d'odeur et de couleur, leur faible toxicité, leur absence d'action corrosive, leur stabilité et leur facilité d'emploi. Leur plus grand désavantage est leur prix, car ils sont plus chers que les solutions d'hypochlorite. Une de leurs 
propriétés, qui peut aussi être un désavantage, dans certaines utilisations, est leur activité superficielle, la production de mousse pouvant quelquefois être gênante. Les C.A.Q. sont aussi incompatibles avec certains détersifs, en particulier les anioniques; mais ceci ne présente pas de difficultés, car il existe d'autres détersifs qui ne sont pas incompatibles aux C.A.Q. La chaleur est évidemment la meilleure méthode de stérilisation, mais elle est quelquefois impraticable ou même indésirable. Du reste, le prix de la vapeur ou de l'eau chaude peut être plus important qu'on ne le suppose généralement. Utilisés correctement, les C.A.Q. et l'hypochlorite donnent des résultats similaires.

La dernière communication a été présentée par C.H. Chalmers, chef de la Section de l'Alimentation et de la Laiterie des Services d'Hygiène de l'Ecosse, sur la «valeur de la vapeur comme agent stérilisant ». La vapeur a, d'après lui, deux propriétés d'une valeur et d'une importance particulières pour la stérilisation du matériel laitier, propriétés qui ne sont pas possédées au même degré par les produits chimiques utilisés actuellement dans les laiteries : l'efficacité de l'action et le pouvoir de pénétration. $C$. expose ensuite les désavantages de l'hypochlorite. Les spores de $B$. cereus et autres mésophiles ne sont pas aisément détruites par les hypochlorites et sont même encore plus résistantes aux quaternaires. La soude caustique utilisée dans le nettoyage par immersion pour la stérilisation des groupes de machines à traire ne détruit pas les spores des organismes aérobies. L'hypochlorite n'a pas une action toxique directe sur les bactéries parce qu'il a d'abord à subir une modification chimique libérant son agent toxique. Cette modification combinée avec le temps pris par l'agent actif pour détruire les organismes signifie pratiquement que la surface à stériliser doit être exposée plus longtemps à l'action du produit chimique que dans le cas de la vapeur, facteur important quand il s'agit de la destruction des spores. En outre, et ceci est aussi important, la proportion de l'agent actif libéré disponible pour la destruction des organismes est modifiée d'une façon importante par la quantité relative d'autres matières organiques existantes exigeant aussi leur part de l'agent actif disponible. Même un petit dépôt de résidus de lait sur la surface réduit la proportion de l'agent actif disponible pour la destruction des bactéries. En fait, ceci ne se produit pas seulement quand il y a du tartre sur la surface à stériliser, mais l'hypochlorite s'unit lui-même au dépôt pour produire un composé qui est très corrosif, même pour l'acier inoxydable. Il en résulte que la surface ne doit pas seulement être entièrement propre, mais que la solution d'hypochlorite doit être soigneusement pré- 
parée pour que la concentration utilisée soit suffisante pour correspondre aux exigences si elle doit être efficace comme stérilisante. Les composés quaternaires ont aussi à subir une modification, avant de pouvoir détruire les bactéries. Cette modification connue sous le nom d'ionisation, a pour effet de les dédoubler en une partie anionique et une partie cationique. Les parties cationiques émigrent vers les bactéries, se concentrent autour d'elles, et procèdent éventuellement à leur destruction. Ceci n'est pas toujours rapide et divers facteurs entrent en jeu qui règlent la vitesse de migration des portions cationiques. Dans la stérilisation pratique du matériel, cette période de ralentissement doit être accordée pour permettre aux quaternaires d'être suffisamment longtemps en contact avec la surface à stériliser. Il faut aussi se rappeler que les portions cationiques émigrent aussi vers toutes les autres matières organiques présentes et que, par conséquent, tous les cations ne sont pas disponibles pour la destruction bactérienne. Ainsi que pour les hypochlorites, il est essentiel de s'assurer que la surface à stériliser est propre et exempte de matière organique étrangère, que la concentration du quaternaire utilisé est mesurée exactement et que le produit chimique reste suffisamment longtemps au contact de la surface, sinon la stérilisation n'est effectuée que partiellement. Les composés quaternaires ont d'autres inconvénients qui les rendent moins convenables dans la pratique que la vapeur comme agents stérilisants. Ils sont moins efficaces que les hypochlorites comme bactéricides; mais il y a lieu aussi de tenir compte de la qualité de l'eau (dureté) et de la nature du détersif quand on utilise le quaternaire avec un tel produit. D'autre part, les quaternaires ont la propriété d'adhérer fortement à la surface du matériel et un rincage soigneux à l'eau stérile est indispensable pour les enlever, sinon le lait peut être infecté. Une telle contamination peut être cancérigène. La vapeur ne crée pas ces difficultés et ces dangers. En outre, elle pénètre dans tous les petits coins, dans toutes les fentes pour les rendre stériles, ce qui n'est pas le cas des bactéricides chimiques. Les parties en caoutchouc sont difficiles à stériliser avec les produits chimiques alors que la vapeur y parvient. Il y a enfin le problème de la corrosion. Quand le matériel métallique est stérilisé à la vapeur, il est élevé à une température telle que toute vapeur condensée est évaporée rapidement et que le matériel est laissé sec. Il n'y a donc pas de corrosion due à la vapeur. Quand on utilise une solution d'un produit chimique, les traces du produit chimique laissées sur le matériel tendent à mettre en route le phénomène de la corrosion. $C$. insiste sur ce point avec beaucoup de détails sans oublier la question de l'allergie 
de certains ouvriers aux produits chimiques. Tous les facteurs étant pris en considération, $C$. se demande s'il y a réellement à choisir entre la vapeur et la stérilisation chimique en ce qui concerne le coût des opérations. Au cours des dernières années, la production de la vapeur a été considérablement simplifiée par l'introduction des producteurs de vapeur fonctionnant électriquement pendant les périodes creuses de la journée à prix réduit. De toutes façons, il faut produire de l'eau chaude car le matériel ne peut pas être lavé efficacement sans utilisation d'un détersif chaud et on sait que les hypochlorites sont plus efficaces comme agents stérilisants quand ils sont utilisés en solution très chaude. De toutes façons, même si les bactéricides chimiques sont utilisés régulièrement, il est convenable, sinon nécessaire, de stériliser périodiquement le matériel à l'eau chaude ou à la vapeur. Pour terminer, $C$. n'affirme pas que la vapeur soit, dans tous les cas, le seul bactéricide satisfaisant, car il y a des cas où il n'est pas indiqué d'utiliser de la vapeur et où il est nécessaire d'utiliser les produits chimiques ou l'eau chaude (à une température d'au moins $85^{\circ}$ ), par exemple, pour la stérilisation des cuves refroidisseuses du lait en vrac à expansion directe, ou des réfrigérants à lait. En somme, les produits chimiques, la vapeur et l'eau chaude ne doivent pas être considérés comme s'opposant mais comme complémentaires. La vapeur possède néanmoins certains caractères qui en font, dans la plupart des cas, le bactéricide de choix.

De la longue discussion générale qui a suivi ces exposés, la "lumière" ne pouvait pas sortir, quoique certains points des exposés des conférenciers aient été clarifiés. Il semble toutefois résulter des débats que la désinfection par la vapeur ou la chaleur est entièrement satisfaisante, mais qu'elle est peut-être trop coûteuse. Beaucoup des mauvais résultats obtenus avec les produits chimiques sont dûs à leur utilisation défectueuse. Ils donnent de bons résultats quand ils sont utilisés convenablement. 